PROCEEDINGS OF THE

AMERICAN MATHEMATICAL SOCIETY

Volume 134, Number 8, Pages 2383-2392

S 0002-9939(06)08258-X

Article electronically published on March 21, 2006

\title{
ON EXTENDED EIGENVALUES AND EXTENDED EIGENVECTORS OF SOME OPERATOR CLASSES
}

\author{
M. T. KARAEV
}

(Communicated by N. Tomczak-Jaegermann)

\begin{abstract}
We give a complete description of the set of extended eigenvectors of the Volterra integration operator $V, V f(x)=\int_{0}^{x} f(t) d t$, on $L^{2}[0,1]$, which strengthens the result of a paper by Biswas, Lambert, and Petrovic (2002). We also introduce the concept of a well splitting operator and study its extended eigenvalues and extended eigenvectors.
\end{abstract}

\section{INTRODUCTION AND PRELIMINARIES}

Let $E$ be a Banach space, and denote by $\mathcal{B}(E)$ the algebra of all bounded linear operators on $E$. If $A$ is an operator in $\mathcal{B}(E)$ and $\lambda$ is a complex number, then following Biswas, Lambert and Petrovic [1] we say that a complex number $\lambda$ is an extended eigenvalue of $A$ if there exists a nonzero operator $X$ in $\mathcal{B}(E)$ such that $A X=\lambda X A$; such an operator $X$ is called extended eigenvector corresponding to $\lambda$. The set of all extended eigenvalues of $A$ will be called the extended point spectrum, and it will be denoted as $\sigma_{p}^{e x t}(A)$. For a given $\lambda \in \sigma_{p}^{e x t}(A)$ we define $\{A\}_{\lambda}^{\prime}$ as the set of all $\lambda$-extended eigenvectors for $A\left(\{A\}_{1}^{\prime}\right.$ is $\{A\}^{\prime}$, the commutant of $A$ ). The set of all extended eigenvectors for $A$ will be denoted as $\{A\}_{\text {ext }}^{\prime}$, i.e., $\{A\}_{\text {ext }}^{\prime}=\bigcup_{\lambda \in \sigma_{p}^{\text {ext }}(A)}\{A\}_{\lambda}^{\prime}$. The basic facts about the extended eigenvalues and extended eigenvectors of operators can be found in [1] -3 .

In what follows, $V$ will denote the simple Volterra integration operator on $L^{2}[0,1]$ defined as

$$
(V f)(x)=\int_{0}^{x} f(t) d t .
$$

It was established in [1] that the set of extended eigenvalues of the Volterra integration operator $V$ is precisely the set $(0, \infty)$. Moreover, it was shown in [1, Theorem 6 , that for each such extended eigenvalue $\lambda$, the appropriate extended eigenvector can be found in the class of integral operators. In other words, for each $\lambda>0$, the equation

$$
X V=\lambda V X
$$

Received by the editors March 3, 2005 and, in revised from, March 14, 2005.

2000 Mathematics Subject Classification. Primary 47A15.

Key words and phrases. Extended eigenvalue, extended eigenvector, Volterra integration operator.

(C)2006 American Mathematical Society 
has a nonzero integral operator as a solution. In this paper, we give a complete description of the set $\{V\}_{\text {ext }}^{\prime}$ of extended eigenvectors of the integration operator $V$ on $L^{2}[0,1]$ (see Theorem 1 in Section 2) which strengthens Theorem 6 of the paper [1. In Section 3 we study the set of extended eigenvalues and extended eigenvectors of so-called well splitting operators on a Banach space $E$. In Section 4 we give an application of Deddens algebras and Shulman subspaces to the "extended" spectral theory.

\section{EXTENDED EIGENVECTORS FOR $V$}

Let $\varphi:[0,1] \rightarrow[0,1]$ be a measurable function. A composition operator $C_{\varphi}$ is defined as $C_{\varphi} f(x)=f(\varphi(x))$. The composition operator $C_{\lambda x}$, where $\lambda \in[0,1]$, will be denoted simply as $C_{\lambda}$. The so-called Duhamel operator $\mathcal{D}_{f}$ on $L^{2}[0,1]$ is defined as

$$
\mathcal{D}_{f} g \stackrel{\text { def }}{=}(f \circledast g)(x) \stackrel{\text { def }}{=} \frac{d}{d x} \int_{0}^{x} f(x-t) g(t) d t .
$$

The following result describes the set $\{V\}_{\text {ext }}^{\prime}$ of Volterra integration operator $V$ on $L^{2}[0,1]$.

Theorem 1. Let $\lambda \in(0, \infty)$ and let $X \in \mathcal{B}\left(L^{2}[0,1]\right)$ be a nonzero operator. Then:

(i) if $\lambda \leq 1$, then $X V=\lambda V X$ if and only if $X=\mathcal{D}_{X 1} C_{\lambda}$, that is,

$$
(X f)(x)=\frac{d}{d x} \int_{0}^{x}(X \mathbf{1})(x-t) f(\lambda t) d t, \quad f \in L^{2}[0,1] ;
$$

(ii) if $\lambda>1$, then $X V=\lambda V X$ if and only if $X C_{1 / \lambda}=\mathcal{D}_{X 1}$.

Proof. (i) Let $X V=\lambda V X$. Then

$$
X V^{n}=\lambda^{n} V^{n} X
$$

for any $n \geq 1$, that is,

$$
X V^{n} f=\lambda^{n} V^{n} X f
$$

for all $f \in L^{2}[0,1]$. In particular,

$$
X V^{n} \mathbf{1}=\lambda^{n} V^{n} X \mathbf{1},
$$

that is,

or

$$
X\left(\frac{x^{n-1}}{(n-1) !} * \mathbf{1}\right)=\lambda^{n} \frac{x^{n-1}}{(n-1) !} * X \mathbf{1},
$$

$$
X \frac{x^{n}}{n !}=\lambda^{n} \frac{x^{n-1}}{(n-1) !} * X \mathbf{1}, \quad n \geq 1 .
$$

* - multiplying the last equality by $\mathbf{1}$ we obtain that

$$
\mathbf{1} * X \frac{x^{n}}{n !}=\lambda^{n}\left(\mathbf{1} * \frac{x^{n-1}}{(n-1) !}\right) * X \mathbf{1},
$$

that is,

for any $n \geq 1$. Hence

$$
\mathbf{1} * X \frac{x^{n}}{n !}=\frac{(\lambda x)^{n}}{n !} * X \mathbf{1},
$$

$$
\mathbf{1} * X p(x)=p(\lambda x) * X \mathbf{1}
$$


for all polynomials $p$. Since $L^{2}[0,1]$ is a Banach algebra with respect to the convolution product $*$, it follows from the last equality that

$$
1 * X f(x)=f(\lambda x) * X \mathbf{1}
$$

for all $f \in L^{2}[0,1]$. Since $V f(x)=\mathbf{1} * f$ for every $f \in L^{2}[0,1]$, the last equality means that

$$
V X f(x)=\mathcal{K}_{X 1} C_{\lambda} f(x),
$$

where $\mathcal{K}_{X 1}$ denotes the usual convolution operator on $L^{2}[0,1], \mathcal{K}_{X 1} g(x) \stackrel{\text { def }}{=} X 1 *$ $g=\int_{0}^{x}(X \mathbf{1})(x-t) g(t) d t, g \in L^{2}[0,1]$. From this, by applying operator $\frac{d}{d x}$ we obtain that

$$
(X f)(x)=\frac{d}{d x} \mathcal{K}_{X \mathbf{1}} C_{\lambda} f(x)
$$

that is,

$$
(X f)(x)=\frac{d}{d x} \int_{0}^{x}(X \mathbf{1})(x-t) f(\lambda t) d t
$$

or

$$
X f=\mathcal{D}_{X 1} C_{\lambda} f
$$

for all $f \in L^{2}[0,1]$, which means that $X=\mathcal{D}_{X 1} C_{\lambda}$. Now it suffices to show that if $X=\mathcal{D}_{X 1} C_{\lambda}$, then $X V=\lambda V X$. In fact, for all $f \in L^{2}[0,1]$ we have

$$
\begin{aligned}
X V f(x) & =\mathcal{D}_{X 1} C_{\lambda} f(x)=\mathcal{D}_{X \mathbf{1}}(V f)(\lambda x) \\
& =X \mathbf{1} \circledast(V f)(\lambda x)=X \mathbf{1} \circledast(\lambda x \circledast f(\lambda x)) \\
& =\lambda x \circledast(X \mathbf{1} \circledast f(\lambda x))=\lambda x \circledast \mathcal{D}_{X \mathbf{1}} C_{\lambda} f(x) \\
& =\lambda\left(x \circledast \mathcal{D}_{X \mathbf{1}} C_{\lambda} f(x)\right)=\lambda V \mathcal{D}_{X \mathbf{1}} C_{\lambda} f(x) \\
& =\lambda V X f(x),
\end{aligned}
$$

which completes the proof of (i).

(ii) Let $X V=\lambda V X$. Clearly $V X=\frac{1}{\lambda} X V$, which implies that $V^{n} X=X\left(\frac{1}{\lambda} V\right)^{n}$ for every $n \geq 1$. The same arguments as in the proof of (i) yield the equality

$$
X f\left(\frac{x}{\lambda}\right)=X \mathbf{1} \circledast f, \quad f \in L^{2}[0,1],
$$

where $\circledast$ is the Duhamel product defined by (1). Conversely, if $X C_{1 / \lambda}=\mathcal{D}_{X 1}$, then since $V f(x)=x \circledast f$ and $\mathcal{D}_{X \mathbf{1}} V=V \mathcal{D}_{X \mathbf{1}}$, for all polynomials $p$ we have

$$
\begin{aligned}
\lambda V X p(x) & =\lambda V X C_{1 / \lambda} C_{\lambda} p(x) \\
& =\lambda V \mathcal{D}_{X 1} C_{\lambda} p(x)=\lambda V \mathcal{D}_{X 1} p(\lambda x) \\
& =\lambda \mathcal{D}_{X 1} V p(\lambda x)=\lambda X C_{1 / \lambda} V p(\lambda x) \\
& =\lambda X C_{1 / \lambda}(x \circledast p(\lambda x))=X C_{1 / \lambda}(\lambda x \circledast p(\lambda x)) \\
& =X C_{1 / \lambda}(V p)(\lambda x)=X V p(x),
\end{aligned}
$$

which completes the proof of (ii) (because the set of polynomials is dense in $L^{2}[0,1]$ ). Theorem 1 is proved.

The following result was proved by a different method in [1], Theorem 7 .

Corollary 2. The composition operator $C_{\varphi}$ satisfies the equation $C_{\varphi} V=\lambda V C_{\varphi}$, where $\lambda \in(0,1]$, if and only if $\varphi(x)=\lambda x, x \in[0,1]$. 
Proof. It is obvious that $C_{\varphi} \mathbf{1}=\mathbf{1}$. Then according to part (i) of Theorem 1 we have that $C_{\varphi} V=\lambda V C_{\varphi}$ if and only if

$$
C_{\varphi} f(x)=\frac{d}{d x} \int_{0}^{x} f(\lambda t) d t
$$

that is,

$$
C_{\varphi} f(x)=f(\lambda x)=C_{\lambda} f(x)
$$

for all $f \in L^{2}[0,1]$, i.e., $C_{\varphi}=C_{\lambda}$, or $\varphi(x)=\lambda x$. This completes the proof.

\section{WELL SPLITTING OPERATORS}

Let $X$ be a separable Banach space and $A \in \mathcal{B}(X)$. An operator $A$ is called a splitting operator in $X$ if for every $x \in X$ there exists a linear densely defined operator $B_{x}$ (generally nonbounded) such that

$$
A^{n} x=B_{x} y_{n}
$$

for every $n, n=0,1,2, \ldots$, and for some complete system $\left\{y_{n}\right\}_{n \geq 0}$ of the space $X$. We say that the splitting operator $A$ is well splitting if for every $x \in X$ the corresponding operators $B_{x}$ in (3) are bounded in $X$. It is immediate from the last definition that a well splitting operator is cyclic if for some $x \in X$ an operator $B_{x}$ has dense range in $X$. It is easy to see that the concept of splitting operator is a generalization of the so-called basis operator introduced by Nikolski 4 .

Example 1. If $X$ denotes any of the spaces $C^{(n)}[0,1]$ and $W_{p}^{(n)}[0,1]$ (Sobolev space), $1 \leq p<+\infty$, then it is not difficult to show that the Volterra integration operator $V$ acting in $X$ is a well splitting operator. Indeed, let us denote by $\circledast$ the Duhamel product defined as

$$
(f \circledast g)(x)=\frac{d}{d x} \int_{0}^{x} f(x-t) g(t) d t
$$

for all $f, g \in X$. Simple calculations show that $X$ is a Banach algebra with respect to the Duhamel product $\circledast$. In particular, for any $f \in X$ the "Duhamel operator" $\mathcal{D}_{f}, \mathcal{D}_{f} g \stackrel{\text { def }}{=} f \circledast g$, is a bounded operator in $X$. Now it follows immediately from (4) that

$$
V^{n} f=\frac{x^{n}}{n !} \circledast f, n \geq 0,
$$

that is,

$$
V^{n} f=\mathcal{D}_{f}\left(\frac{x^{n}}{n !}\right), n \geq 0,
$$

for all $f \in X$. By Weierstrass's approximation theorem $\left\{\frac{x^{n}}{n !}\right\}_{n \geq 0}$ is a complete system in $X$, and hence, formula (5) means that $V$ is a well splitting operator in $X$.

In what follows, for every complete system $\left\{y_{n}\right\}_{n \geq 0}$ in $X$ we will denote by the symbol $\Lambda_{\left\{y_{n}\right\}}$ the set of all complex numbers $\lambda$ for which the diagonal operator $D_{\{\lambda\}}, D_{\{\lambda\}} y_{n} \stackrel{\text { def }}{=} \lambda^{n} y_{n}, n \geq 0$, is bounded in $X$.

The following result gives, in particular, more general examples of well splitting operators. 
Theorem 3. Let $X$ be a Banach space with basis $\left\{e_{n}\right\}_{n \geq 0}$, and T, Te $e_{n}=\lambda_{n} e_{n+1}$, $\lambda_{n} \neq 0, n \geq 0$, be a weighted shift operator continuously acting in $X$. Let us set $w_{n} \stackrel{\text { def }}{=} \lambda_{0} \lambda_{1} \ldots \lambda_{n-1}, w_{0} \stackrel{\text { def }}{=} 1$. Suppose that the following conditions are satisfied:

1) There exists an integer $N \geq 0$ such that

$$
\sum_{n, m \geq N}\left|\frac{w_{n+m}}{w_{n} w_{m}}\right|<+\infty
$$

2) $\left\|e_{n+m}\right\| \leq c\left\|e_{n}\right\|\left\|e_{m}\right\|$ for all $n, m \geq 0$, and for some number $c>0$. Then:

(i) $T$ is well splitting operator on a $X$;

(ii) If $\lambda \in \Lambda_{\left\{e_{n}\right\}}$ is a nonzero number and $A \in \mathcal{B}(X)$ is a nonzero operator, then $A \in\{T\}_{\lambda}^{\prime}$ if and only if $A D_{\{\lambda\}}=\mathcal{D}_{A e_{0}}$.

Proof. For the arbitrarily chosen elements $x=\sum_{n \geq 0} x_{n} e_{n}$ and $y=\sum_{n \geq 0} y_{n} e_{n}$ of the space $X$, let us define a discrete analogy of the Duhamel product by the following formula:

$$
x \circledast y \stackrel{\text { def }}{=} \sum_{n, m \geq 0} \frac{w_{n+m}}{w_{n} w_{m}} x_{n} y_{m} e_{n+m} .
$$

By virtue of the conditions of theorem, formula (6) is correctly defined. By setting $\tau_{n}(x) \stackrel{\text { def }}{=} \sum_{k \geq n} x_{k} e_{k}$ and using the conditions of the theorem we have:

$$
\begin{aligned}
x \circledast y= & \sum_{n, m \geq 0} \frac{w_{n+m}}{w_{n} w_{m}} x_{n} y_{m} e_{n+m} \\
= & \sum_{n \geq 0} \frac{x_{n}}{w_{n}} \sum_{m \geq 0} \frac{w_{n+m}}{w_{m}} y_{m} e_{n+m} \\
= & x_{0} \sum_{m \geq 0} y_{m} e_{m}+\frac{x_{1}}{w_{1}} \sum_{m \geq 0} \frac{w_{m+1}}{w_{m}} y_{m} e_{m+1} \\
& +\frac{x_{2}}{w_{2}} \sum_{m \geq 0} \frac{w_{m+2}}{w_{m}} y_{m} e_{m+2}+\ldots \\
& +\frac{x_{N-1}}{w_{N-1}} \sum_{m \geq 0} \frac{w_{m+N-1}}{w_{m}} y_{m} e_{m+N-1} \\
& +\sum_{n \geq N} \frac{x_{n}}{w_{n}} \sum_{m \geq 0} \frac{w_{n+m}}{w_{m}} y_{m} e_{n+m} \\
= & x_{0} y+\frac{x_{1}}{w_{1}} T y+\ldots+\frac{x_{N-1}}{w_{N-1}} T^{N-1} y \\
& +y_{0} \tau_{N}(x)+\frac{y_{1}}{w_{1}} \tau_{N}(T x)+\ldots+\frac{y_{N-1}}{w_{N-1}} \tau_{N}\left(T^{N-1} x\right) \\
& +\sum_{n \geq N} \sum_{m \geq N} \frac{w_{n+m}}{w_{n} w_{m}} x_{n} y_{m} e_{n+m} .
\end{aligned}
$$


Hence

$$
\begin{aligned}
\|x \circledast y\| \leq & \left|x_{0}\right|\|y\|+\left|\frac{x_{1}}{w_{1}}\right|\|T y\|+\ldots+\left|\frac{x_{N-1}}{w_{N-1}}\right|\left\|T^{N-1} y\right\| \\
& +\left|y_{0}\right|\left\|\tau_{N}(x)\right\|+\left|\frac{y_{1}}{w_{1}}\right|\left\|\tau_{N}(T x)\right\|+\ldots+\left|\frac{y_{N-1}}{w_{N-1}}\right|\left\|\tau_{N}\left(T^{N-1} x\right)\right\| \\
& +\sum_{n \geq N} \sum_{m \geq N}\left|\frac{w_{n+m}}{w_{n} w_{m}}\right|\left|x_{n}\right|\left|y_{m}\right|\left\|e_{n+m}\right\| \\
\leq & \widetilde{c}\|x\|\|y\|+c \sum_{n \geq N} \sum_{m \geq N}\left|\frac{w_{n+m}}{w_{n} w_{m}}\right|\left|x_{n}\right|\left\|e_{n}\right\|\left|y_{m}\right|\left\|e_{m}\right\| \\
\leq & C\|x\|\|y\|,
\end{aligned}
$$

that is,

$$
\|x \circledast y\| \leq C\|x\|\|y\|
$$

for all $x, y \in X$. It follows from (6) and (7) that $X$ is a Banach algebra with respect to $\circledast$ and with unit $e_{0}$. For every $x \in X$ we define the following operator:

$$
\mathcal{D}_{x} y=x \circledast y, y \in X .
$$

It is clear from (6) that

$$
T^{n} y=w_{n} e_{n} \circledast y
$$

for every $y \in X$ and any $n \geq 0$, that is,

$$
T^{n} y=\mathcal{D}_{y}\left(w_{n} e_{n}\right) .
$$

In fact, for every $y \in X$ and any $n \geq 0$ we have

$$
\begin{aligned}
T^{n} y & =T^{n}\left(\sum_{m \geq 0} y_{m} e_{m}\right)=\sum_{m \geq 0} y_{m} T^{n} e_{m} \\
& =\sum_{m \geq 0} y_{m} \lambda_{m} \lambda_{m+1} \ldots \lambda_{m+n-1} e_{m+n}=\sum_{m \geq 0} y_{m} \frac{w_{m+n}}{w_{m}} e_{m+n} \\
& =\sum_{m \geq 0} w_{n} y_{m} \frac{w_{n+m}}{w_{n} w_{m}} e_{n+m}=\sum_{m \geq 0} w_{n} y_{m}\left(e_{n} \circledast e_{m}\right) \\
& =w_{n} e_{n} \circledast \sum_{m \geq 0} y_{m} e_{m}=w_{n} e_{n} \circledast y=\mathcal{D}_{y}\left(w_{n} e_{n}\right),
\end{aligned}
$$

as desired. Hence, formula (8) means that $T$ is a well splitting operator in $X$.

(ii) Clearly, if $\lambda A T=T A$, then $\lambda^{n} A T^{n}=T^{n} A, n \geq 0$. In particular,

$$
A \lambda^{n} T^{n} e_{0}=T^{n} A e_{0}, n \geq 0 .
$$

Using formula (8), from this we obtain that

$$
A w_{n} \lambda^{n} e_{n}=A\left(w_{n} \lambda^{n} e_{n} \circledast e_{0}\right)=\left(w_{n} e_{n} \circledast A e_{0}\right)
$$

or

that is,

$$
A \lambda^{n} e_{n}=\left(e_{n} \circledast A e_{0}\right),
$$

$$
A \lambda^{n} e_{n}=A e_{0} \circledast e_{n}, n \geq 0 .
$$

From this

$$
A P_{\lambda}=A e_{0} \circledast P
$$


for all polynomials $P=\sum_{n \geq 0} P_{n} e_{n} \in X$, where $P_{\lambda} \stackrel{\text { def }}{=} \sum_{n \geq 0} \lambda^{n} P_{n} e_{n}=D_{\{\lambda\}} P$, that is,

$$
A D_{\{\lambda\}} P=A e_{0} \circledast P .
$$

Since $(X, \circledast)$ is a Banach algebra (see inequality $(7)$ ), from this we deduce that

$$
A D_{\{\lambda\}} x=A e_{0} \circledast x
$$

for all $x \in X$, i.e.,

$$
A D_{\{\lambda\}}=\mathcal{D}_{A e_{0}},
$$

where $\mathcal{D}_{A e_{0}}$ is the Duhamel operator.

Conversely, let us prove that every nonzero operator $A$ satisfying (10) belongs to $\{T\}_{\lambda}^{\prime}$. Indeed, for all vector polynomials $P=\sum_{m=0}^{\operatorname{deg} P} P_{m} e_{m}$ we have

$$
\begin{aligned}
T A P & =T A D_{\{\lambda\}} D_{\left\{\frac{1}{\lambda}\right\}} P=T A D_{\{\lambda\}} P_{\left\{\frac{1}{\lambda}\right\}}=T \mathcal{D}_{A e_{0}} P_{\left\{\frac{1}{\lambda}\right\}} \\
& =w_{1} e_{1} \circledast \mathcal{D}_{A e_{0}} P_{\left\{\frac{1}{\lambda}\right\}}=\mathcal{D}_{w_{1} e_{1}} \mathcal{D}_{A e_{0}} P_{\left\{\frac{1}{\lambda}\right\}}=\mathcal{D}_{A e_{0}} \mathcal{D}_{w_{1} e_{1}} P_{\left\{\frac{1}{\lambda}\right\}} \\
& =\mathcal{D}_{A e_{0}}\left(w_{1} e_{1} \circledast P_{\left\{\frac{1}{\lambda}\right\}}\right)=\mathcal{D}_{A e_{0}} \lambda\left(\frac{w_{1} e_{1}}{\lambda} \circledast P_{\left\{\frac{1}{\lambda}\right\}}\right) \\
& =\lambda \mathcal{D}_{A e_{0}}\left(\frac{w_{1} e_{1}}{\lambda} \circledast P_{\left\{\frac{1}{\lambda}\right\}}\right)=\lambda A D_{\{\lambda\}}\left(\frac{w_{1} e_{1}}{\lambda} \circledast \sum_{m=0}^{\operatorname{deg} P} P_{m} \frac{1}{\lambda^{m}} e_{m}\right) \\
& =\lambda A D_{\{\lambda\}}\left[\frac{1}{w_{1}} \frac{w_{1}}{\lambda} \sum_{m=0}^{\operatorname{deg} P} \frac{w_{m+1}}{w_{m}} \frac{P_{m}}{\lambda^{m}} e_{m+1}\right] \\
& =\lambda A D_{\{\lambda\}}\left[\frac{1}{\lambda} \sum_{m=0}^{\operatorname{deg} P} \lambda_{m} \frac{P_{m}}{\lambda^{m}} e_{m+1}\right] \\
& =\lambda A D_{\{\lambda\}}\left[\frac{1}{\lambda} \sum_{m=0}^{\operatorname{deg} P} \lambda \lambda_{m} \frac{P_{m}}{\lambda^{m+1}} e_{m+1}\right] \\
& =\lambda A D_{\{\lambda\}}\left[\sum_{m=0}^{\operatorname{deg} P} \lambda_{m} P_{m} \frac{1}{\lambda^{m+1}} e_{m+1}\right] \\
& =\lambda A D_{\{\lambda\}}\left[D_{\left\{\frac{1}{\lambda}\right\}} \sum_{m=0}^{\operatorname{deg} P} \lambda_{m} P_{m} e_{m+1}\right] \\
& =\lambda A D_{\{\lambda\}} D_{\left\{\frac{1}{\lambda}\right\}} \sum_{m=0}^{\operatorname{deg} P} P_{m} e_{m} \\
& =\lambda A T P,
\end{aligned}
$$

and so $T A x=\lambda A T x$ for all $x \in X$, that is, $A \in\{T\}_{\lambda}^{\prime}$. The theorem is proved.

Remark 1. It can be proved that if $\lambda \in \Lambda_{\left\{e_{n}\right\}}$, then $T A=\lambda A T$ if and only if

$$
\mathcal{D}_{A x}=A \mathcal{D}_{x} D_{\{\lambda\}} \text {. }
$$

Indeed, if $T A=\lambda A T$, then we obtain that

$$
\begin{aligned}
A \mathcal{D}_{x} D_{\{\lambda\}} w_{n} e_{n} & =A \mathcal{D}_{x} \lambda^{n} w_{n} e_{n}=A\left(x \circledast \lambda^{n} w_{n} e_{n}\right) \\
& =A\left(\lambda^{n} T^{n} x\right)=T^{n} A x=w_{n} e_{n} \circledast A x=\mathcal{D}_{A x} w_{n} e_{n},
\end{aligned}
$$

for all $n \geq 0$, which obviously implies (11). 
Conversely, if an operator $A$ has the property (11), then we have

$$
\begin{aligned}
A T x & =A\left(w_{1} e_{1} \circledast x\right)=A \mathcal{D}_{x} w_{1} e_{1}=\frac{1}{\lambda} A \mathcal{D}_{x} w_{1} \lambda e_{1} \\
& =\frac{w_{1}}{\lambda} A \mathcal{D}_{x} D_{\{\lambda\}} e_{1}=\frac{w_{1}}{\lambda} \mathcal{D}_{A x} e_{1}=\frac{1}{\lambda} \mathcal{D}_{A x} w_{1} e_{1} \\
& =\frac{1}{\lambda}\left(A x \circledast w_{1} e_{1}\right)=\frac{1}{\lambda} T A x
\end{aligned}
$$

for all $x \in X$, and hence $T A=\lambda A T$, which completes the proof.

Our next result shows that the property (11) is shared by any well splitting operator.

Theorem 4. Let $T$ be a well splitting operator on a separable Banach space $X$ defined by a complete system $\left\{y_{n}\right\}_{n \geq 0}$, i.e.,

$$
T^{n} x=B_{x} y_{n}, n \geq 0,
$$

for each $x \in X$. Let $\lambda \in \Lambda_{\left\{y_{n}\right\}}$ be any nonzero number. Then $\lambda A T=T A$ if and only if

$$
B_{A x}=A B_{x} D_{\{\lambda\}}
$$

for each $x \in X$.

Proof. Let $T$ be a well splitting operator on $X$, and let $A$ be a bounded linear operator on $X$ such that $\lambda A T=T A$. Then it is obvious that

$$
\begin{aligned}
B_{A x} y_{n} & =T^{n} A x=A\left(\lambda^{n} T^{n}\right) x=A B_{x} \lambda^{n} y_{n} \\
& =A B_{x} D_{\{\lambda\}} y_{n}
\end{aligned}
$$

for each $x \in X$ and $n$. Since the system $\left\{y_{n}\right\}_{n>0}$ is complete in $X$, it follows that $B_{A x}=A B_{x} D_{\{\lambda\}}$ for each $x \in X$. Conversely, if an operator $A \in \mathcal{B}(X)$ satisfies $B_{A x}=A B_{x} D_{\{\lambda\}}$ for each $x \in X$, then we have that $B_{A x} y=A B_{x} D_{\{\lambda\}} y$ for each $y \in X$; in particular, $B_{A x} y_{1}=A B_{x} D_{\{\lambda\}} y_{1}$. Then we have

$$
\begin{aligned}
T A x & =B_{A x} y_{1}=A B_{x} D_{\{\lambda\}} y_{1}=A B_{x} \lambda y_{1} \\
& =\lambda A B_{x} y_{1}=\lambda A T x,
\end{aligned}
$$

for each $x \in X$, so it follows that $T A=\lambda A T$, that is, $A \in\{T\}_{\lambda}$, as desired. The proof is completed.

Corollary 5. $T A=A T$ if and only if $B_{A x}=A B_{x}$ for each $x \in X$.

\section{An application of Deddens algebras and Shulman subspaces}

In this short section Deddens operator algebras and Shulman subspaces (which were introduced in [5]) are used for the investigation of extended eigenvalues of operators. Throughout the text, $H$ will signify a Hilbert space of generally unspecified dimension. The following definition is due to Deddens [6] (see [5] and 7] for general definitions).

Definition 1. Let $A$ be invertible in $\mathcal{B}(H)$ :

$$
\mathcal{B}_{A} \stackrel{\text { def }}{=}\left\{X \in \mathcal{B}(H):\left\|A^{n} X A^{-n}\right\| \text { bounded for } n \geq 0\right\} \text {. }
$$


Thus, $\mathcal{B}_{A}$ is the collection of operators having bounded conjugation orbits, conjugation being by $A$. In general, Deddens algebra $\mathcal{B}_{A}$ clearly contains $\{A\}^{\prime}$ and is an algebra because

$$
\begin{aligned}
\left\|A^{n} X_{1} X_{2} A^{-n}\right\| & =\left\|A^{n} X_{1} A^{-n} A^{n} X_{2} A^{-n}\right\| \\
& \leq\left\|A^{n} X_{1} A^{-n}\right\|\left\|A^{n} X_{2} A^{-n}\right\| .
\end{aligned}
$$

In [6, $\mathcal{B}_{A}$ was introduced as an alternative for the description of nest algebras, and this result suggests that the boundedness condition defining $\mathcal{B}_{A}$ is of interest for any invertible $A$.

For two operators $L, M \in \mathcal{B}(H)$ let us denote by $\mathcal{U}(L, M)$ the Shulman subspaces of the space $\mathcal{B}(H)$ (see [5]), defined by

$$
\mathcal{U}(L, M) \stackrel{\text { def }}{=}\{L\}^{\prime}+\{L\}^{\prime} M .
$$

Such subspaces have been studied in detail by Shulman in relation with nontransitivity of root algebras (see [8, 9]).

The relation between Deddens algebras and Shulman subspaces is established in the next theorem. (Below the number $\lambda \in \mathbb{C}$ is assumed to be such that $L_{\lambda} \stackrel{\text { def }}{=}$ $\lambda I+L$ is an invertible operator.)

Theorem 6 ([5]). Let the operators $L, M \in \mathcal{B}(H)$ satisfy the Kleinecke-Shirokov condition, i.e., $X \stackrel{\text { def }}{=}[M, L] \in\{L\}^{\prime}$. Then the intersection of Deddens algebra $\mathcal{B}_{\lambda I+L}$ and the weak closure of Shulman subspace $\mathcal{U}(L, M)$ coincide with a commutant of the operator $L$, that is,

$$
\mathcal{B}_{\lambda I+L} \cap \overline{\mathcal{U}}(L, M)^{w}=\{L\}^{\prime} .
$$

In particular, since $[T, V]=V^{2}$, we have

$$
\mathcal{B}_{I+V} \cap \overline{\mathcal{U}(V, T)}^{w}=\{V\}^{\prime},
$$

where $T$ is the multiplication operator in $L^{2}[0,1],(T f)(x)=x f(x)$.

Here we prove the following theorem.

Theorem 7. Let $\lambda$ be a nontrivial scalar in the unit circle $\mathbb{T}$ of a complex plane, i.e., $\lambda \in \mathbb{T} \backslash\{1\}$, and let $A \in \mathcal{B}(H)$ be an invertible operator satisfying the KleineckeShirokov condition for some operator $M \in \mathcal{B}(H)$. Suppose that $\mathcal{B}_{A^{-1}} \subseteq \overline{\mathcal{U}}(A, M)^{w}$. If $\lambda X A=A X$, then $X=0$.

Proof. Let $\lambda X A=A X$. Then $\lambda^{n} X A^{n}=A^{n} X, n \geq 0$. From this $A^{n} X A^{-n}=$ $\lambda^{n} X, n \geq 0$, and hence, $\left\|A^{n} X A^{-n}\right\|=\|X\|, n \geq 0$, which means that $X \in \mathcal{B}_{A}$. On the other hand, since $X A=\frac{1}{\lambda} A X$, we have that $X A^{n}=\frac{1}{\lambda^{n}} A^{n} X, n \geq 0$, from which it follows that $A^{-n} X A^{n}=\frac{1}{\lambda^{n}} X, n \geq 0$, that is, $\left\|A^{-n} X A^{n}\right\|=\|X\|$, $n \geq 0$, which implies that $X \in \mathcal{B}_{A-1}$. Since $\mathcal{B}_{A^{-1}} \subseteq \overline{\mathcal{U}}(A, M)^{w}$, we have that $X \in \overline{\mathcal{U}}(A, M)^{w}$. Thus, $X \in \mathcal{B}_{A} \cap \overline{\mathcal{U}}(A, M)^{w}=\{A\}^{\prime}$ (see Theorem 6), which yields that $(\lambda-1) A X=0$, that is, $X=0$ as desired. The theorem is proved.

Corollary 8. Let $N \in \mathcal{B}(H)$ be a square-zero operator satisfying the KleineckeShirokov condition for some operator $M \in \mathcal{B}(H)$. If $\lambda \in \mathbb{T} \backslash\{1\}$, and $\lambda X(I+N)=$ $(I+N) X$, then $X=0$. 
Proof. It is known (see, for instance, [5, 6]) that $\mathcal{B}_{(I+N)^{-1}}=\mathcal{B}_{I-N}=\{N\}^{\prime} \subset$ $\overline{\mathcal{U}}(I+N, M)^{w}$, that is, the operator $I+N$ satisfies condition of Theorem 7 , and the desired result is obtained.

I thank the referee for a number of important remarks.

\section{REFERENCES}

[1] A. Biswas, A. Lambert and S. Petrovic, Extended eigenvalues and the Volterra operator, Glasgow Math. J. 44(2002), 521-534. MR.1956558 (2004c:47039)

[2] A. Biswas, A. Lambert and S. Petrovic, On the extended eigenvectors for operators, Preprint.

[3] A. Lambert, Hyperinvariant subspaces and extended eigenvalues, New York J. Math. 10 (2004), 83-88. MR2052366 (2004k:47007)

[4] N. K. Nikolski, Basisness and unicellularity of weighted shift operators, Izvestiya Akad. Nauk SSR, Ser. Mat., 32 (1968), 1123-1137 (in Russian). MR0238098 (38:6374)

[5] M. T. Karaev and H. S. Mustafayev, On some properties of Deddens algebras, Rocky Mountain J. Math., 33, 3(2003), 915-926. MR2038531 (2005f:47153)

[6] J. A. Deddens, Another description of nest algebras, Lecture Notes in Math., Vol. 693, Springer, New York, 1978, 77-86. MR0526534 (80f:47033)

[7] M. T. Karaev and S. Pehlivan, Some results for quadratic elements of a Banach algebra, Glasgow Math. J. 46(2004), 431-441. MR2094801

[8] V. S. Shulman, On transitivity of some space of operators, Functional Anal. Appl., 16(1982), 91-92. MR0648826 (83d:47015)

[9] V. S. Shulman, Invariant subspace and spectral mapping theorem, Banach Center Publ., Vol. 30, PWN, Warsaw, 1994, 313-325. MR1285617 (95m:47008)

Department of Mathematics, Suleyman Demirel University, 32260 Isparta, Turkey

E-mail address: garayev@fef.sdu.edu.tr 